\title{
The Korean urban rural elderly cohort study: study design and protocol
}

\author{
Eun Young Lee ${ }^{1}$, Hyeon Chang Kim², Yumie Rhee ${ }^{1}$, Yoosik Youm ${ }^{3}$ Kyoung Min Kim, Ju Mi Lee², Dong Phil Choi², \\ Young Mi Yun ${ }^{2}$ and Chang Oh Kim ${ }^{1 *}$
}

\begin{abstract}
Background: Korea is one of the fastest aging countries and is expected to become a super-aged society within 12 years. The Korean Urban Rural Elderly (KURE) study was developed to evaluate the epidemiological characteristics and establish the prevention and management of major disorders of the elderly in Korea.

Methods/Design: The KURE study is a community-based prospective cohort study on health, aging, and common geriatric disorders of Korean elderly persons aged at least 65 years. To construct a cohort reflecting both urban and rural areas, we selected 2 representative communities in the country. To establish multidisciplinary approaches to geriatric health, this study was performed by researchers in the divisions of geriatrics, preventive medicine, endocrinology, and sociology. The baseline examinations began in 2012; the study will follow more than 4,000 elderly Koreans over 10 years. The first and second follow-up health examinations will be performed every 4 years. Every 2 years after each health examination, inter-assessment interview will be conducted to improve participant retention.

Discussion: The KURE study will provide longitudinal epidemiologic data on health, aging, and common geriatric disorders of the elderly in Korea. This is a comprehensive, multidisciplinary study of the elderly with respect to biological, physical, socio-economic, and environmental factors. The results of this study will contribute to improve public health and welfare policies for the aging society in Korea.
\end{abstract}

Keywords: Aging, Elderly, Cohort, Longitudinal study, Health

\section{Background}

Worldwide population aging has accelerated since the 1980 s, with more than $11 \%$ of the world's population already over the age of 60 years in 2010 [1]. The elderly population in Korea has increased with an unprecedented rate and is expected to be more than $20 \%$ of the total population in 2026 , namely a super-aged society. This accelerated aging of the population may lead to a rapid increase in socioeconomic burden as well as medical costs. In Korea, the aging index ([population over the age of 65 years] $\div$ [population under the age of 15 years] $\times 100$ ) increased from $11.2 \%$ in 1980 to $68.4 \%$ in 2010. During the same period, the elderly dependency ratio ([population

\footnotetext{
* Correspondence: cokim@yuhs.ac

${ }^{1}$ Department of Internal Medicine, Yonsei University College of Medicine, 50-1 Yonsei-ro, Seodaemun-gu, Seoul, Korea

Full list of author information is available at the end of the article
}

over the age of 65 years] $\div$ [population aged $15-64$ years] $\times$ 100 ) increased from $6.1 \%$ to $15.2 \%$. The elderly dependency ratio is expected to be 20.0 in 2018, classifying as an aged society [2]. This suggests that the magnitude of the elderly population that depends on young adults will increase about 2-fold from 2000 to 2018 .

In addition to aging itself, disability-adjusted life expectancy is also emerging as an important factor for public health in Korea. In 2005, disability-adjusted life expectancies in Korea were 67.5 years in men and 69.6 years in women, about 10 years shorter than the life expectancy in the same year. Many comorbidities occurring with aging have a great effect on disability-adjusted life expectancy. In addition, these comorbidities are characterized by multifaceted factors including biological, physical, psychosocial, and environmental factors. Therefore, we developed the Korean Urban Rural Elderly (KURE) study, a prospective 
longitudinal cohort of the elderly population in Korea, to obtain comprehensive data on health, aging, and prevalent disorders. The aim of this article is to describe the design of the KURE study.

\section{Methods/Designs Study design and participants}

This is a 12-year prospective cohort study of communitydwelling elderly persons aged 65 years and older living in urban or rural areas in Korea. To reflect the characteristics of the elderly residing in urban and rural areas, 3 administrative districts, called "Gu," were selected as an urban area and one administrative district, called "Do," was selected as a rural area. These areas were selected on the basis of their elderly populations and accessibility to the research center. Eligibility criteria, presented in Table 1, were chosen to maximize recruitment of community-dwelling elderly persons. Participants are mainly recruited by 1 of the 4 following methods: (1) recruiters visiting a random street and house, (2) participants visiting local government health facilities, (3) participants contact after seeing promotional poster for the study, or (4) acquaintances of study participants. During health examinations, participants undergo a thorough physical examination, face-to-face interview, and laboratory tests. Information gathered from interviews includes demographic, physical/clinical, nutrition, life style, psychological, daily activity, and social support and network. After the baseline health examination, participants will be taken full health examination every 4 years including face-to-face interview. Additional follow-up interviews will be performed between heath examinations (Figure 1). Participants will be followed for at least 10 years, or until death. To reduce losses to follow-up, a brief telephone interview is scheduled for the years when a full health examination is conducted and 2 years later. If initial contact by telephone fails, we will re-contact participants up to 5 times during different days and times of day. During the telephone interviews, information including change in residence, use of health services in the past year, and mortality is recorded. Furthermore, participants will be invited to free lectures on health and receive newsletters and holiday cards annually.

\section{Sample size calculations}

To evaluate the relevance of the proposed sample size ( $n=4,000)$, we estimated the minimum detectable odds ratio between an outcome and an exposure. We assumed the exposure frequency to be $25 \%$ and $33 \%$, respectively. Prevalence of outcome (5-year mortality) was assumed with a range of $1 \%, 2 \%, 4 \%$, and $16 \%$. Power and a 2 -sided alpha were set a priori at $90 \%$ and 0.05 , respectively. Based on the $33 \%$ exposure frequency, the minimum detectable odds ratio was estimated to range from 2.33 for an outcome prevalence of $1 \%$ to 1.31 for an outcome prevalence of $16 \%$. Based on the $25 \%$ of exposure frequency, the minimum detectable odds ratio was estimated to range from 2.44 for an outcome prevalence of $1 \%$ to 1.34 for an outcome prevalence of $16 \%$.

\section{General health and medical history}

Participants receive a comprehensive physical examination and complete a questionnaire by face-to-face interview (Table 2). All interviewers are trained for at least 2 days before administering the survey to participants. Medical information includes current and past medical history, family history, medication, subjective health status, vaccination, incontinence symptoms, falling, fracture, pain, and sleep. Chronic disease diagnoses by a physician are investigated by self-report using a list of 16 diseases including cardiovascular and respiratory disease, cerebrovascular attack, diabetes, thyroid disorders, arthritis, cataract, depression, and cancer. Consistent with the Korean National Health and Nutrition Examination Survey, all questionnaires about chronic disease consist of diagnosis time and process of treatment [3]. Smoking and alcohol consumption are also investigated using the questionnaire.

\section{Blood pressure and pulse rate}

Blood pressure and pulse rate are measured using an electronic manometer (HEM-7111, Omron Healthcare Co., Ltd., Kyoto, Japan). Blood pressure and pulse rate are measured at heart-level with a standardized cuff. Values are recorded after 5 minutes of rest in the sitting position and repeated after another 5 minutes of rest. If blood pressure is significantly different between the first and the second measurements, a third measurement is taken

Table 1 Eligibility criteria for subject selection into the Korean urban rural elderly cohort study

\begin{tabular}{ll}
\hline Inclusion criteria & Exclusion criteria \\
\hline - Age $\geq 65$ years & - Age $<65$ years \\
- Living in the study area: 3 northwestern districts of Seoul (Mapo-gu, & - Living in the current residence less than 8 months of a calendar year \\
Seodaemun-gu, and Eunpyeong-gu) and 1 rural area of Incheon & • Planning to move from the current residence within 2 years \\
(Ganghwa county) & \\
- Capable of communication with the research team & \\
- Provided written informed consent & \\
\hline
\end{tabular}


RECRUITMENT
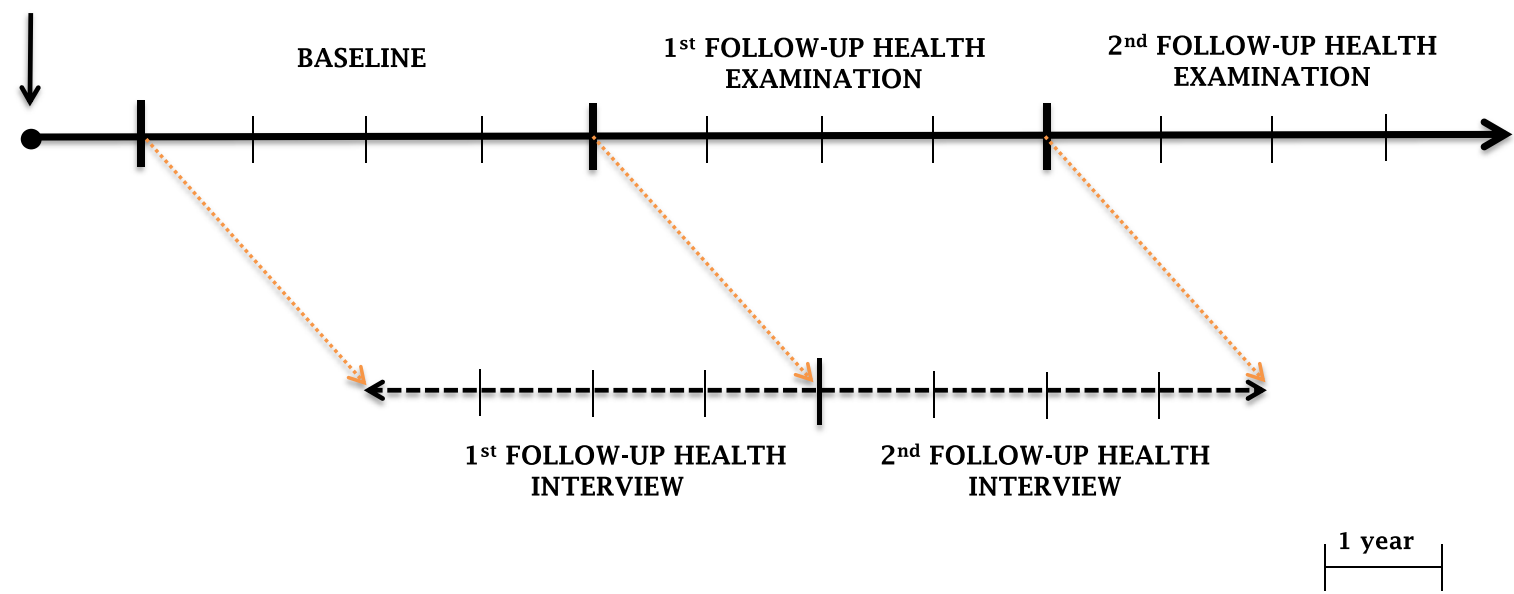

Figure 1 Conduct of the study.

after 5 minutes of rest. Radial arterial pulse waves are simultaneously measured noninvasively using an applanation tonometry and central blood pressure is calculated by the HEM-9000AI (Omron Healthcare Co., Ltd., Kyoto, Japan).

\section{Anthropometric measurements}

During anthropometric measurements, all participants are asked to wear light clothing without shoes or jewelry. Height is measured within $0.1 \mathrm{~cm}$ using a stadiometer (DS-102, JENIX, Korea) with an upright position. Body weight is determined within $0.1 \mathrm{~kg}$ using an electronic scale (DB-150, CAS, Korea). Each device is calibrated prior to each examination. Body mass index $\left(\mathrm{kg} / \mathrm{m}^{2}\right)$ is calculated as weight in kilograms divided by the square of height in meters. Circumference of the waist, hip, thigh, and calf are measured within $0.1 \mathrm{~cm}$ using a plastic tapeline. Waist circumference is measured over the midpoint between the lower border of the ribs and iliac crest in the mid-axillary plane. Hip circumference is measured over the longest point of the buttocks. Thigh circumference is measured at the midpoint between the inguinal crease and proximal border of the patella. Calf circumference is measured at the largest point of the calf.

\section{Physical activities and function}

Physical activity is assessed by a self-report scale: (1) nonactive (mostly resting or lying down), (2) less active (mostly sitting without exercise), (3) active (mostly sitting but with regular light exercise, commuting, housework, standing at work, etc.), (4) very active (mostly standing or vigorous exercise). In addition, exercise is assessed according to the intensity, frequency, and average time.

The chair-rise test, a sit-to-stand test repeated 5 times, is performed by participants who are able to sit and stand. During the test, participants are instructed to do

Table 2 Overview of the demographic and general health assessment in the Korean urban rural elderly cohort study

\begin{tabular}{|c|c|}
\hline Classification & Measures and instruments \\
\hline General health and medical history & $\begin{array}{l}\text { Current and past medical history, family history, subjective health status, vaccination, } \\
\text { incontinence symptoms, falling, fracture, pain, smoking, alcohol, sleep }\end{array}$ \\
\hline Anthropometry & Height; weight; body mass index; circumference of waist, hip, thigh, and calf \\
\hline Physical activities and function & Exercise (intensity and frequency), timed up and go test, grip strength, chair-rise test \\
\hline Activities of daily living & Korean activities of daily living scale, Korean instrumental activities of daily living scale \\
\hline Depression & $\begin{array}{l}\text { Five Geriatric Depression Scale from the Korean version of comprehensive } \\
\text { geriatric assessment, Korean version of the Geriatric Depression Scale - Short Form }\end{array}$ \\
\hline Cognitive function & Korean version of Mini-Mental State Examination for dementia screening \\
\hline Nutritional status and weight & Mini-nutrition assessment, loss of appetite, changes of body weight within the prior 3 months \\
\hline Socioeconomic status & Education, occupation, household income, family income, marital status \\
\hline Social support & $\begin{array}{l}\text { Social network (size, range, homophily, density, degree centrality, closeness, } \\
\text { betweenness, walk betweenness, brokerage, and overlapping), stressful life events }\end{array}$ \\
\hline
\end{tabular}


their best and the total time taken to complete the test is recorded in seconds. Timed up and go test is conducted on a leveled walking surface. At the beginning of the test, participants get up from a chair, walk $3 \mathrm{~m}$ at their regular walking speed, turn, walk back, and sit down again. Investigators record the total time from getting up to sitting down in seconds using a stopwatch. Grip strength is measured by a dynamometer in kilograms with participants' arms resting on a desk with the elbow angled properly. Participants hold a dynamometer for as long as they can for a maximum of 3 seconds, and then relax their grip. This test is conducted in each of the hands and repeated after 20 seconds rest.

\section{Activity of daily living}

Functional status of daily life is assessed using 2 types of questionnaires: the Korean version of activities of daily living [4] and a modified Korean version of instrumental activities of daily living [5-7]. The Korean version of activities of daily living is composed of 6 basic items such as bathing, dressing, feeding, rising or lying down, walking, and toileting. The modified Korean version of instrumental activities of daily living consists of 8 items such as shopping, using a phone, using public transportation, doing light house work, doing laundry, preparing meals, taking medicine, and managing money. The ability to perform each task is rated according to the degree of dependency on another person. These questionnaires validated among an elderly population $[4,6]$.

\section{Depression and cognitive function}

Depressive symptoms are assessed in 2 steps. First, participants are asked 5 questions about depressive symptoms using the Geriatric Depression Scale from Korean version of the comprehensive geriatric assessment [8]. The second step uses the Korean version of the Geriatric Depression Scale - Short Form $[9,10]$, if participants answer "yes" to at least 1 of the 5 questions in the first step. The Korean version of the Geriatric Depression Scale - Short Form consists of 15 questions originating from the Geriatric Depression Scale with 30 items [11]; each question yields a score from 0 to 3 and the total score is used to evaluate depression as follows: depression (scores >10), moderate depressive symptom (scores from 6 to 9), and normal (scores from 0 to 5 ).

We administered the Korean version of the Mini-Mental State Examination for dementia screening to evaluate cognitive function and screen for dementia, which contains 30 items with scores ranging from 0 to 30 and includes areas of memory; attention; language; calculation; visuospatial abilities; and orientation to time, place, and person [12]. A total score of less than 24 indicates cognitive dysfunction. Recently, a total score of 27 has been suggested to detect mild cognitive impairment, especially among the elderly [13].

\section{Nutritional status and weight}

Nutritional status is assessed by the Mini Nutritional Assessment, which consists of 6 questions on food intake and weight loss in the last 3 months, mobility, psychological stress or acute illness in the last 3 months, neuropsychological problems, and body mass index or calf circumference. Nutritional status was defined as follows: normal (scores from 12 to 14 points), at risk of malnutrition (scores from 8 to 11 points), and malnourished (scores from 0 to 7 points). Weight loss is classified as intended or unintended weight loss. Unintentional weight loss is related to a decrease in function and mobility in the elderly [14].

\section{Socioeconomic status and social support}

Socioeconomic factors are assessed with regard to educational, occupational, financial, and marital status. Detailed data on socioeconomic status are composed of the highest level and duration of education, current and most longterm occupation, and subjective and objective financial status. Evaluation on social support is focused on social network as well as stressful life events. On the basis of the frequency, size, and closeness of social contact, social network is assessed to provide several parameters as follows: network size, range, homophily, density, degree of centrality, closeness, betweenness, walk betweenness, brokerage, and overlapping [15]. Participants are also asked whether they lived alone or with someone else (a spouse, children, or others) in the face-to-face interview. Previous studies have reported that social support was associated with beneficial effects on cardiovascular, endocrine, and immune systems and, furthermore, might regulate these physiologic responses in elderly [16].

\section{Blood and urine analysis}

After an overnight fast, blood and urine samples are collected in the morning. Blood samples are centrifuged for 10 minutes at $3000 \mathrm{rpm} 30$ minutes after sampling. All samples are delivered to the Seoul Clinical Laboratories (Seoul, Korea) for analysis within 24 hours. As shown in Table 3, biochemical tests include complete blood count, kidney and liver function test, lipid profile, endocrine function, inflammatory markers, and urinalysis. Residual samples are stored at $-80^{\circ} \mathrm{C}$ for future analysis.

\section{Bone mineral density and fracture risk assessment}

The bone mineral density of the lumbar spine (L1-L4) and total hip are measured using dual-energy X-ray absorptiometry (Hologic QDR 4500A, Waltham, USA). The average precision error of this machine is less than $1.1 \%$. All scans are performed by a trained operator on the same day as the health examination. Vertebral fracture assessment by dual-energy X-ray absorptiometry was performed to evaluate fracture in the spine. Fracture risk assessment was also calculated using the FRAX ${ }^{\circledast}$ model for South Korea [17]. 
Table 3 Laboratory and biochemical tests administered in the Korean urban rural elderly cohort study

\begin{tabular}{|c|c|c|}
\hline \multirow{2}{*}{\multicolumn{2}{|c|}{$\begin{array}{l}\text { Classification } \\
\text { Complete blood count }\end{array}$}} & Measures \\
\hline & & $\begin{array}{l}\text { White blood cell, white blood cell differential, red blood cell, hemoglobin, hematocrit, } \\
\text { mean corpuscular volume, mean corpuscular hemoglobin, mean corpuscular } \\
\text { hemoglobin concentration, red cell distribution width, platelet count }\end{array}$ \\
\hline \multicolumn{2}{|l|}{ Renal panel } & Blood urea nitrogen, creatinine \\
\hline \multicolumn{2}{|l|}{ Liver function test } & $\begin{array}{l}\text { Total protein, albumin, alkaline phosphatase, aspartate aminotransferase, } \\
\text { alanine aminotransferase, total bilirubin }\end{array}$ \\
\hline \multicolumn{2}{|l|}{ Lipid profile } & Total cholesterol, triglyceride, high density lipoprotein cholesterol \\
\hline \multicolumn{2}{|l|}{ Carotid ultrasonography } & Carotid intima-media thickness, presence of plaque \\
\hline \multirow[t]{3}{*}{ Endocrine } & Glucose metabolism & Glucose, glycated hemoglobin, insulin \\
\hline & Bone metabolism & Calcium, phosphorus, parathyroid hormone, 25-hydroxyvitamin D, osteocalcin \\
\hline & Thyroid function & $\begin{array}{l}\text { Triiodothyronine, free thyroxine, thyroid stimulating hormone, } \\
\text { thyroglobulin antibodies, thyroid peroxidase antibodies }\end{array}$ \\
\hline \multicolumn{2}{|l|}{ Inflammation } & Erythrocyte sedimentation rate, high sensitivity C-reactive protein \\
\hline \multicolumn{2}{|l|}{ Others } & Ferritin, uric acid \\
\hline \multicolumn{2}{|l|}{ Urinalysis } & $\begin{array}{l}\text { Microalbumin, pH, nitrite, specific gravity, protein, glucose, ketone, } \\
\text { bilirubin, blood, urobilinogen, red blood cells, white blood cells, } \\
\text { epithelial cells, casts, bacteria, crystals, color }\end{array}$ \\
\hline \multicolumn{2}{|l|}{ Bone mineral density } & Dual energy $\mathrm{X}$-ray absorptiometry \\
\hline \multicolumn{2}{|l|}{ Electrocardiography } & 12-channel electrocardiography \\
\hline \multicolumn{2}{|l|}{ Physical activity } & Accelerometry \\
\hline \multicolumn{2}{|l|}{ Body composition analysis } & Bioimpedence test \\
\hline
\end{tabular}

\section{Carotid intima-media thickness (IMT) and plaque}

Intima-media thickness (IMT) and plaques in both carotid arteries are assessed using high-resolution B-mode ultrasonography with an 8-MHz linear probe (L5-13IS, Samsung Medicine, Co., Ltd., Seoul, Korea). According to a predetermined, standardized protocol [18], carotid IMT is measured at the distal common carotid artery, $1 \mathrm{~cm}$ proximal to the carotid bulb, and the proximal internal carotid arteries from transverse and longitudinal orientations by an experienced technician. The IMT is defined as the distance between the adventitia-media interface and intima-lumen interface. The mean and maximum values of 6 measurements from the bilateral IMTs are used in the analyses. Carotid plaque is defined as the focal lesion protruding into the arterial lumen at least $0.5 \mathrm{~mm}$ or $50 \%$ greater than surrounding IMT value.

\section{Accelerometry}

Accelerometers (ActiGraph GT3X-pus, ActiGraph Co., Pensacola, FL) are used to objectively measure physical activity and sleep time. Participants are instructed to wear the accelerometer on the wrist of their non-dominant arm for 7 consecutive days for 24 hours a day except during water activities, and to log wearing time. Physical activities are recorded using both 10 - and 60 -second intervals. At least 1 valid day of accelerometer wearing is to be included in the analysis, with a valid day defined as wearing the accelerometer for at least 10 hours in a 24-hour period. Accelerometry includes various parameters including step count, total activity counts, metabolic equivalent of task, and sleeping time. Accelerometers have been found useful for objectively measuring physical activity and to predict functional decline in community-dwelling elderly persons [19].

\section{Bioimpedence}

To assess body composition, bioimpedence is measured using the InBody 720 (Biospace Co., Ltd., Seoul, Korea) with alternating currents of $250 \mathrm{~mA}$ and 6 frequencies $(1 \mathrm{kHz}, 5 \mathrm{kHz}, 50 \mathrm{kHz}, 250 \mathrm{kHz}, 500 \mathrm{kHz}$, and $1 \mathrm{MHz}$ ) at 5 locations: right arm, left arm, trunk, right leg, and left leg. According to the manufacturer's instructions, bioimpedence is measured in the morning and before eating, exercising, or bathing. With light cloths and no accessories, participants should stand barefoot on the foot electrodes and hold the hand electrodes. Arms and legs should be spread out so not to contact each other and other parts of body. Participants had to maintain this posture during the test. Bioimpedence analysis is a non-invasive, easy, and an inexpensive method for assessing body composition and is applicable to a wide range of subjects. In addition, bioimpedence has been shown to be a valid method for measuring body composition and visceral fat area in the elderly [20-22].

\section{Data collection and statistical analysis}

All data are collected and managed using the database program (iCReaT, Korea National Institutes of Health, Korea Centers for Disease Control and Prevention). Data 
are verified by double checking for erroneous or missing values. Only trained and authorized persons are able to access the database system. All participants are interviewed with structured questionnaires, which are designed for this study. Interviewers record responses on paper forms; all information is kept in a locked space. Secondary data will be collected from national registries to obtain information on the use of health services and mortality. Trained health investigators and medical laboratory technicians perform physical examination and laboratory tests, respectively. They are instructed to follow the standardized clinical protocol.

Data will be presented as mean \pm standard deviations, percentages, odds rations (for cross-sectional analysis) or relative risks (for longitudinal analysis). The prevalence of various geriatric disease and related-factors will be estimated according to age, gender, and areas. Outcomes will include mortality, newly-developed diseases or disabilities and any other health issues related with the elderly. Student t-test or analysis of variance will be used to compare variables among the different groups. To investigate the associations between outcomes and studied parameters, multivariate analysis will be used. Possible confounding factors, which are found in univariate analysis, as well as clinically relevant parameters will be adjusted in multivariate analysis. The level of $P$ value less than 0.05 will be considered as statistical significance.

\section{Ethics}

The study protocol was approved by the ethics committee of Severance Hospital. Participants provided written informed consent (including permission to use secondary data such as register data on health service use) before enrolling in the study and completing the first examination. Participants were informed that they could chose to withdraw from the study at any time. Interviews were performed in a separate room to protect participants' privacy. According to the International Conference on Harmonization Good Clinical Practice guidelines, all information recorded was anonymized.

\section{Discussion}

This study protocol describes the design and methods used in the KURE study. The KURE cohort study is designed to assess health status, aging, and major geriatric diseases in community-dwelling elderly persons in Korea. In this study, we focus on not only epidemiologic characteristics of geriatric disorders, but also the relationship between social support (network) and geriatric health in terms of cardiovascular, neuroendocrine, and immune system. Hence, this study is able to evaluate the impact of the social environment of the elderly on their health and quality of life in addition to elucidating biological risk factors of geriatric disease.
There are several strengths in this study. First, this is the cohort study for long follow-up time. Therefore, this study may assist in determining causal associations between predicted determinants of health and outcomes and be used to obtain trajectories of geriatric health over time [23,24]. Second, this study has a large sample size, with a statistical power to detect mild to moderate associations. Third, the face-to-face interview method may be especially useful in obtaining accurate information, especially among the elderly who may not immediately understand or response the questionnaire. Fourth, this study recruited the elderly from both urban and rural areas. This will help us investigate geriatric health in different geographic and socioeconomic environments and life styles. Lastly, the KURE investigative team is multidisciplinary including experts in geriatrics, preventive medicine, infection and immunology, endocrinology, and sociology. This team approach may be helpful in developing a unique research resource for a multifaceted understanding of geriatric health. The major limitation of this study is to recruit only voluntary participants. This might cause selection bias and not completely represent the whole geriatric population in Korea.

The KURE study will contribute to establishing how various factors such as biological, physical, socio-economical, and environmental factors affect geriatric health and disease by providing comprehensive epidemiologic data of geriatric health in Korea. This will not only improve the understanding of disease prevalence in the elderly, but also assist in preventing disease, and ultimately improving the health management system in Korea.

\section{Abbreviations}

IMT: Intima media thickness; KURE: Korean urban rural elderly.

\section{Competing interests}

The authors declare that they have no competing interests.

\section{Authors' contributions}

EYL contributed to the drafting and revision of the manuscript, supervision of the study, and acquisition of data. HCK contributed to the revision of the manuscript, design/supervision of the study, and statistical methodology. YR contributed to the revision of the manuscript and design/supervision of the study. YY contributed to the revision of the manuscript and design/ supervision of the study with specific focus on social network. KMK contributed to the revision of the manuscript and supervision of the study. JML contributed to the revision of the manuscript, design/ supervision of the study, and acquisition of data. DPC contributed to the revision of the manuscript, supervision of the study, and acquisition of data. YMY contributed to the revision of the manuscript and acquisition/management of data. COK contributed to the revision of the manuscript and design/supervision of the study. All authors read and approved the final manuscript.

\section{Acknowledgements}

The present study is supported by a fund (2012-E63001-001, 2013-E63007-00) by Research of Korea Centers for Disease Control and Prevention.

\section{Author details}

${ }^{1}$ Department of Internal Medicine, Yonsei University College of Medicine, 50-1 Yonsei-ro, Seodaemun-gu, Seoul, Korea. ${ }^{2}$ Department of Preventive Medicine, Yonsei University College of Medicine, 50-1 Yonsei-ro, Seodaemun-gu, Seoul, Korea. ${ }^{3}$ Department of Sociology, Yonsei University, 50 Yonsei-ro, Seodaemun-gu, Seoul, Korea. ${ }^{4}$ Department of Internal Medicine, Seoul National University Bundang Hospital, Seongnam, Korea. 
Received: 5 February 2014 Accepted: 7 March 2014

Published: 19 March 2014

\section{References}

1. World Population Ageing Report: World Population Ageing Report; 2013. http://www.un.org/en/development/desa/population/publications/ageing/ WorldPopulationAgeingReport2013.shtml.

2. Population Projections for Korea: Population Projections for Korea: 2010-2060 (Based on the 2010 Census). http://kosis.kr/ups/ups_01List01.jsp?grp_no=\&pubcode=PJ.

3. Lee HS, Hwang IY, Park YJ, Yoon SH, Han K, Son JW, Ko SH, Park YG, Yim HW: Prevalence, awareness, treatment and control of hypertension in adults with diagnosed diabetes: the Fourth Korea National Health and Nutrition Examination Survey (KNHANES IV). J Hum Hypertens 2013, 27(6):381-387.

4. Choi SH, Na DL, Lee BH, Kang SJ, Ha C, Han S, Erzigkeit H: Validation of the Korean version of the Bayer activities of daily living scale. Hum Psychopharmacol 2003, 18(6):469-475

5. Won CW, Yang KY, Rho YG, Kim SY, Lee EJ, Yoon JL, Cho KH, Shin HC, Cho BR, Oh JR: The development of Korean activities of daily living (K-ADL) and Korean instrumental activities of daily living (K-IADL) scale. J Korean Geriatr Soc 2002, 6(2):107-120.

6. Won CW, Rho YG, SunWoo D, Lee YS: The validity and reliability of Korean instrumental activities of daily living (K-IADL) scale. J Korean Geriatr Soc 2002, 6(4):273-280

7. Lawton MP, Brody EM: Assessment of older people: self-maintaining and instrumental activities of daily living. Gerontologist 1969, 9(3):179-186.

8. Jung S-Y, Kwon IS, Cho B, Yoon JL, Rho YG, Lee E, Won CW, Choi Y-H, Woo DS, Park B-J: Reliability and validity of Korean brief comprehensive geriatric assessment questionnaire. J Korean Geriatr Soc 2006, 10(2):67-76.

9. Sheikh Jl, Yesavage J: Clinical Gerontology: a Guide to Assessment and Intervention. New York: The Harworth Press; 1986.

10. Kee BS: A preliminary study for the standardization of geriatric depression scale short form-Korea version. J Korean Neuropsychiatr Assoc 1996, 35:298-307.

11. Yesavage JA, Brink TL, Rose $T L$, Lum O, Huang $V$, Adey M, Leirer VO: Development and validation of a geriatric depression screening scale: a preliminary report. J Psychiatr Res 1983, 17(1):37-49.

12. Kim TH, Jhoo JH, Park JH, Kim JL, Ryu SH, Moon SW, Choo IH, Lee DW, Yoon JC, Do YJ: Korean version of mini mental status examination for dementia screening and its' short form. Psychiatry Investig 2010, 7(2):102-108.

13. O'Bryant SE, Humphreys JD, Smith GE, Ivnik RJ, Graff-Radford NR, Petersen RC, Lucas JA: Detecting dementia with the mini-mental state examination in highly educated individuals. Arch Neuro/ 2008, 65(7):963-967.

14. Ritchie CS, Locher JL, Roth DL, McVie T, Sawyer P, Allman R: Unintentional weight loss predicts decline in activities of daily living function and life-space mobility over 4 years among community-dwelling older adults. J Gerontol A-Biol 2008, 63(1):67-75.

15. Nettleton DF: Data mining of social networks represented as graphs. Computer Science Review 2013, 7:1-34.

16. Uchino BN, Holt Lunstad J, Uno D, Betancourt R, Garvey TS: Social support and age-related differences in cardiovascular function: an examination of potential mediators. Ann Behav Med 1999, 21(2):135-142.

17. WHO Fracture Risk Assessment Tool. http://www.shef.ac.uk/FRAX/tool.aspx? country $=25$.

18. de Groot E, van Leuven SI, Duivenvoorden R, Meuwese MC, Akdim F, Bots ML, Kastelein JJ: Measurement of carotid intima-media thickness to assess progression and regression of atherosclerosis. Nat Clin Pract Cardiovasc Med 2008, 5(5):280-288.

19. Davis MG, Fox KR: Physical activity patterns assessed by accelerometry in older people. Eur J Appl Physiol 2007, 100(5):581-589.

20. Kyle UG, Genton L, Karsegard L, Slosman DO, Pichard C: Single prediction equation for bioelectrical impedance analysis in adults aged 20-94 years. Nutrition 2001, 17(3):248-253.

21. Demura S, Sato S: Prediction of visceral fat area in Japanese adults: proposal of prediction method applicable in a field setting. Eur J Clin Nutr 2006, 61(6):727-735.

22. Kim M, Kim H: Accuracy of segmental multi-frequency bioelectrical impedance analysis for assessing whole-body and appendicular fat mass and lean soft tissue mass in frail women aged 75 years and older. Eur $J$ Clin Nutr 2013, 67(4):395-400.
23. Karunananthan S, Wolfson C, Bergman H, Béland F, Hogan D: A multidisciplinary systematic literature review on frailty: overview of the methodology used by the Canadian initiative on frailty and aging. BMC Med Res Methodol 2009, 9(1):68.

24. Guralnik JM, Kritchevsky SB: Translating research to promote healthy aging: the complementary role of longitudinal studies and clinical trials. J Am Geriatr Soc 2010, 58(s2):S337-S342.

doi:10.1186/1471-2318-14-33

Cite this article as: Lee et al:: The Korean urban rural elderly cohort study: study design and protocol. BMC Geriatrics 2014 14:33.

\section{Submit your next manuscript to BioMed Central and take full advantage of:}

- Convenient online submission

- Thorough peer review

- No space constraints or color figure charges

- Immediate publication on acceptance

- Inclusion in PubMed, CAS, Scopus and Google Scholar

- Research which is freely available for redistribution 